\title{
Utilização da Arquitetura de Computação em Névoa e Estações Agrometeorológicas para o acompanhamento do clima na lavoura
}

\author{
Mahuan Capeletto Abdala \\ Universidade Estadual do Oeste do Paraná \\ Foz do Iguaçu/PR, Brasil \\ abdalamahuan@gmail.com
}

\author{
Antonio Marcos Massao Hachisuca \\ Universidade Estatual do Oeste do Paraná \\ Foz do Iguaçu/PR, Brasil \\ shiro.foz@gmail.com
}

\begin{abstract}
Resumo- A agricultura moderna exige o aumento da produção de alimentos para suprir as demandas do crescimento da população mundial. Para este objetivo, novas tecnologias e soluções estão sendo aplicadas neste domínio, através da coleta e processamento de informações para aumentar significativamente a produtividade. Nos últimos anos, a evolução tecnológica, permitiu a produção de componentes eletrônicos como sensores e micro controladores a um custo acessível, permitindo que novos conceitos fossem criados, dentre eles destaca-se a Internet das Coisas (Internet of Things - IoT). Através do uso de diversos sensores distribuídos pela lavoura é possível monitorar diferentes variáveis como solo, clima e a própria cultura, porém, o monitoramento remoto das lavouras é um grande desafio tecnológico, pois existe a necessidade de transmitir, armazenar e processar grandes volumes de dados gerados pela rede de sensores, assim como possuir viabilidade econômica para a sua utilização em larga escala e em pequenas propriedades. Para auxiliar no tratamento desse grande volume de dados no local da aplicação é necessário adicionar uma camada de poder computacional entre os dispositivos IoT e a nuvem. Nesse sentido surge um novo conceito chamado computação em névoa, este trata de realizar análise, armazenamento e processamento dos dados na borda da rede. Neste contexto está o projeto SmartFarm, que se baseia no desenvolvimento de solução de hardware e software para a construção de uma rede de sensores agrícolas (AioT). Essa arquitetura possibilita ao produtor e/ou pesquisadores acompanhar os diversos estádios da planta remotamente e em tempo real auxiliando-os na tomada de decisão. Dessa forma para o desenvolvimento de uma plataforma de comunicação, armazenamento e préprocessamento de dados, foi utilizado um Raspberry Pi 3 modelo $B$ atuando como névoa, protocolo de comunicação MQTT (Message Queuing Telemetry Transport) e uma API desenvolvida em Node.js para o fornecimento das informações para o usuário.
\end{abstract}

Keywords- Internet das Coisas, Computação em Névoa, Smart Farms.

\section{INTRODUÇÃO}

O Brasil nas últimas cinco décadas se tornou um dos mais importantes produtores e exportadores mundiais de alimentos, contribuindo com a alimentação de aproximadamente 1,5 bilhão de pessoas no mundo. Esse cenário contribui para o crescimento do País elevando a renda e a geração de empregos, impulsionando a participação da agricultura no Produto Interno Bruto (PIB) brasileiro. (1). O agronegócio no Brasil representa cerca de $23 \%$ do total do PIB brasileiro (2), sendo um importante setor para a economia de nosso país.
Um fator importante que deve ser levado em consideração é o crescimento populacional. Segundo (3) a população mundial chegará a 8,6 bilhões de pessoas em 2030, aproximadamente 9,8 bilhões de pessoas em 2050 e chegue perto dos 11,2 bilhões de habitantes em 2100 , juntamente com este crescimento, ocorre o aumento de expectativa de vida da população mundial. A previsão para 2030 diz que mulheres viverão em média 85,3 anos e homens 78,1 (4).

Neste contexto destaca-se também a expansão da renda per capita da classe média mundial e a manutenção da migração rural-urbana, nos próximos 20 anos. Estes fatores indicam grande potencial de crescimento na demanda por produtos agropecuários para alimentação, ao mesmo tempo em que representa uma grande oportunidade para o Brasil, este por sua vez, é um dos únicos países onde ainda existem áreas para expansão do cultivo, além de possuir clima e território favorável, o que possibilita um aumento de produtividade. Entretanto, a escassez de mão de obra e o acesso a tecnologias pelas pequenas propriedades, em especial a agricultura familiar, serão desafios para que o Brasil consiga aproveitar esta oportunidade (5).

Nos últimos anos, a evolução tecnológica, permitiu a produção de componentes eletrônicos como sensores e microcontroladores a um baixo custo, neste sentido, houve uma popularização de tecnologias como a Internet, redes sem fio e Smartphones. Esta popularização permitiu que novos conceitos fossem criados, dentre eles destaca-se a Internet das Coisas (IoT - do inglês Internet of Things), segundo (6), a "Internet das Coisas" se refere a uma revolução tecnológica que em breve conectará equipamentos como eletrodomésticos, meios de transporte, roupas e maçanetas, todavia, este conceito não se aplica somente a área urbana, na agricultura pode auxiliar à aumentar significativamente sua produtividade, diminuindo o impacto ambiental e reduzindo o desperdício, através do uso de redes de sensores com monitoramento constantemente das condições e necessidades das plantas, animais e insumos da propriedade (7).

A modernização dos processos produtivos, a utilização de sensores e equipamentos para o monitoramento e acompanhamento das lavouras de forma remota e em tempo real, tornam-se necessidades estratégicas para que o Brasil consiga aumentar a produtividade, minimizar o impacto ambiental e, consequentemente, obter maiores retornos financeiros, aumentando a competitividade no mercado mundial (8). 
O monitoramento remoto das lavouras é um grande desafio tecnológico, pois existe a necessidade de transmitir, armazenar e processar grandes volumes de dados (Big data) gerados pela rede de sensores, assim como possuir viabilidade econômica para a sua utilização em larga escala e em pequenas propriedades. $\mathrm{O}$ processamento e gerenciamento desse enorme volume de dados é um desafio para as plataformas e metodologias tradicionais. Normalmente o conjunto de dados contém dados referentes as espécies das plantas, padrões de plantios e safras, parâmetros climáticos, condições ambientais, tipos de solos nutrientes do solo, informações geográficas, registros das colheitas e dados das máquinas (9).

Para auxiliar no tratamento desse grande volume de dados no local da aplicação é necessário adicionar uma camada de poder computacional entre os dispositivos IoT e a nuvem (figura 1), neste sentido surge um novo conceito chamado computação em névoa, este, trata de realizar análise, armazenamento e processamento dos dados na borda da rede (10).

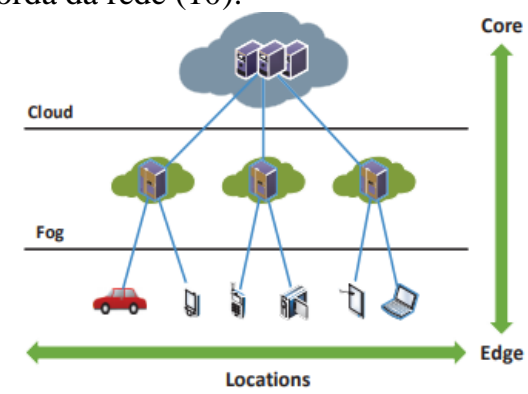

Figura 1. Representação Computação em névoa (11).

No conjunto, a automação, agricultura de precisão e Tecnologia da Informação e Comunicação (TIC), aliadas tornam-se de grande importância no apoio a tomada de decisão para planejamento, monitoramento e predição de riscos na produção (12).

Neste contexto, é criado o projeto SmartFarm (figura 2), o qual se baseia no desenvolvimento de solução de hardware e software que integram sensores climáticos, hídricos, comunicação sem fio e protocolos de comunicação para a construção de uma rede de sensores agrícolas (AioT). Essa arquitetura possibilita o produtor e/ou pesquisadores a acompanhar os diversos estádios fenológicos da planta remotamente e em tempo real facilitando na tomada de decisão.

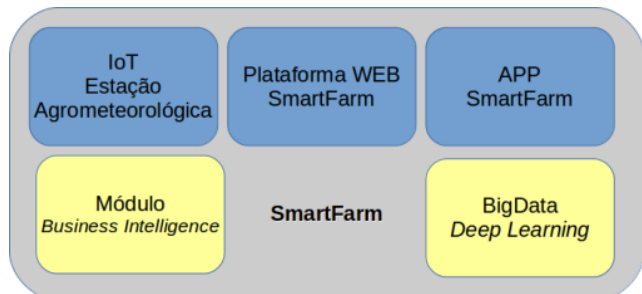

Figura 2. Arquitetura/Módulos da Plataforma SmartFarm

Este trabalho tem como objetivo utilizar o conceito de computação em névoa para o desenvolvimento de uma plataforma de comunicação, armazenamento e préprocessamento dos dados provenientes de uma rede de sensores e estações agrometeorológicas distribuídas pela lavoura, dispostos em uma arquitetura IoT, este projeto será integrado no projeto piloto SmartFarm.

\section{MATERIAIS E MÉTODOS}

As condições climáticas e a disponibilidade hídrica durante o cultivo são fatores que influenciam diretamente na produção, dessa forma, a utilização de tecnologias através do monitoramento remoto das diferentes zonas de manejo da lavoura, torna-se boa prática para o aumento da eficiência produtiva da propriedade rural.

Com o objetivo de realizar estudos e análises do comportamento do clima, planta e solo, para auxiliar o produtor na tomada de decisão, são instaladas estações agrometeorológicas (figura 3) em diferentes pontos da lavoura, as quais possuindo sensores como: temperatura e umidade do ar, chuva, temperatura e umidade do solo a 5 , 20 e $30 \mathrm{~cm}$ de profundidade, pressão atmosférica e luminosidade, além das informações sobre a tensão da bateria e o RSSI do sinal wifi.

Foram instaladas onze estações identificadas como sendo do número 20 ao 30, em uma área de 17.9 ha $^{-1}$ (figura 4) no município de Céu Azul/PR.

O solo da região é classificado Latossolo Vermelho Distroférrico típico (13), e os talhões vem sendo cultivados com sistema de plantio direto a pelo menos 10 anos, com culturas comerciais de soja, milho e trigo em sistema de sucessão de culturas.

A região possui classificação de clima - Cfa - Clima subtropical; temperatura média no mês mais frio inferior a $18^{\circ} \mathrm{C}$ (mesotérmico) e temperatura média no mês mais quente acima de $22^{\circ} \mathrm{C}$, com verões quentes, geadas pouco frequentes e tendência de concentração das chuvas nos

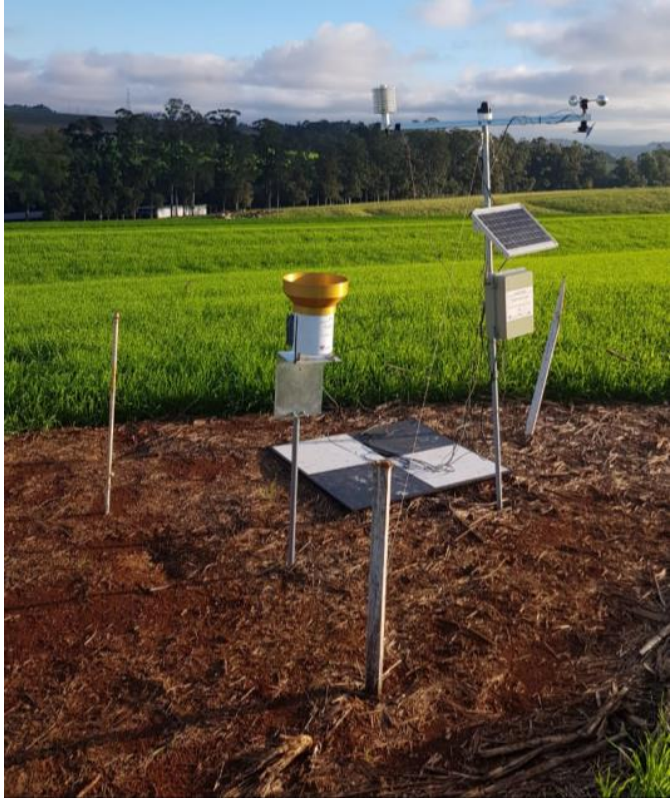

Figura 3. Estação agrometeorológica. 


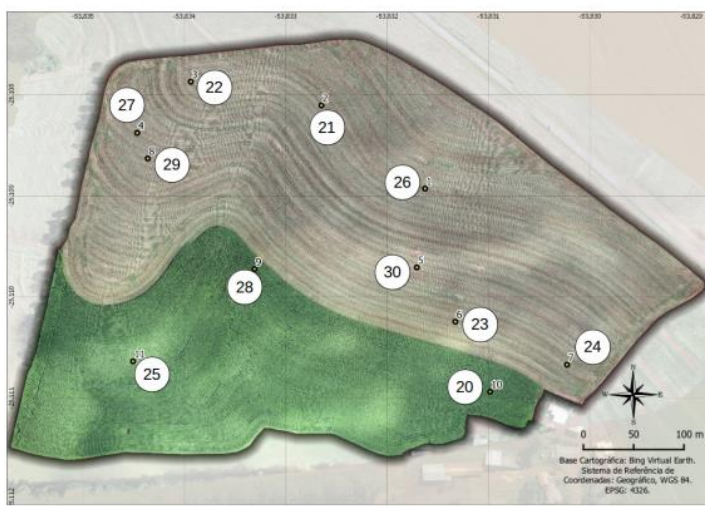

Figura 4. Localização da área de implantação e posições das estações instaladas. (14).

meses de verão, contudo sem estação seca definida

Para a construção da arquitetura de computação em névoa foi utilizado um Raspberry Pi 3 modelo $\mathrm{B}$, trata-se de um microcomputador do tamanho de um cartão de crédito, possui um processador Cortex-A53 64 bit, com 4 núcleos, clock de $1.2 \mathrm{Ghz}, 1 \mathrm{~GB}$ de memória RAM e com o padrão wireless 802.11 n já embutido. O Raspberry Pi foi responsável por fornecer os serviços de préprocessamento, armazenamento e comunicação próximo aos dispositivos, ou seja, no local da aplicação, atuando como servidor local sendo o broker MQTT da solução na camada da névoa. Como broker foi utilizado o Mosca sendo implementado em Node.js.

A comunicação entre as estações e o Raspberry Pi se dá através do protocolo MQTT sob uma rede wireless utilizando uma CPE TP-Link TL-WA7210N, projetada especificamente para a aplicação, o link de internet da propriedade é de $2 \mathrm{MB}$. A estação mais distante, fica aproximadamente $485 \mathrm{~m}$ de distância da antena emissora.

Sob a arquitetura proposta foram desenvolvidas duas APIs (Application Programming Interface) de coleta e manipulação dos dados, as quais atuam na nuvem e possuem como objetivo fornecer comunicação, além de processar e armazenar as informações vindas da névoa, manipular as informações das estações agrometeorológicas, seus sensores e as leituras desses sensores, além de disponibilizar todas essas informações para o usuário.

A figura 5 apresenta a arquitetura proposta para a solução. As estações enviam os dados dos sensores através de uma rede wireless utilizando o protocolo MQTT para o servidor local, ao receber os dados a névoa os armazena localmente e realiza o envio para o servidor na nuvem através da arquitetura REST no formato JSON, auxiliando no tratamento do grande volume de dados e proporcionando melhor integridade e disponibilidade sobre os dados capturados pelos sensores das estações agrometeorológicas.

A utilização da tecnologia em névoa permite reduzir o fluxo de dados para a nuvem, além de ser independente de internet, a qual está sujeita a quedas momentâneas por estar inserida em área rural e sofrer aos fatores externos que influenciam na qualidade da rede.
Caso o Raspberry Pi não possua internet os dados continuam sendo armazenados até que ocorra a confirmação de envio para a nuvem, neste cenário a névoa mantém seu funcionamento normalmente mascarando a falta de internet para os dispositivos.

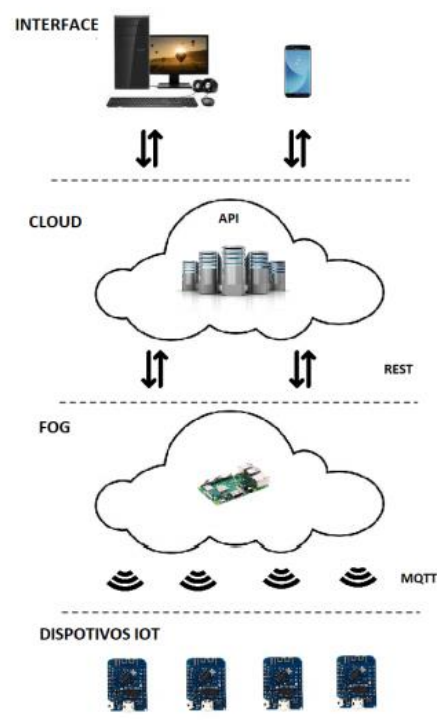

Figura 5. Arquitetura da solução.

Ambas as APIs desenvolvidas, API de coleta e a API de manipulação das informações, atuam no servidor na nuvem. A API de coleta foi desenvolvida com o objetivo de receber os dados vindos da névoa, armazená-los e realizar o processamento desses dados. Já a outra API tem como objetivo disponibilizar o gerenciamento e acompanhamento das informações do sistema pelo usuário, permitindo a manipulação das informações sobre as estações, sensores, as leituras desses sensores e estatísticas do dia como média, máxima e mínima do dia.

\section{RESUlTADOS E DisCUSSÕES}

A figura 6 apresenta um exemplo de resposta a requisição sobre a última leitura de temperatura do ar para a estação 23.

Os funcionamentos das aplicações juntamente com o Raspberry $\mathrm{Pi}$ em testes realizados em laboratório mostraram-se eficientes, não demonstrando qualquer problema, porém, a partir da instalação em campo, foram identificados alguns problemas que foram resolvidos em seguida. Inicialmente todos os dados coletados eram armazenados localmente pelo Raspberry $\mathrm{Pi}$, porém, ao realizar o processamento para enviar os dados para a nuvem notou-se um atraso significativo que aumentava conforme o crescimento da base de dados.

Além do atraso no envio das informações notou-se que estava ocorrendo replicação de dados no servidor na nuvem, acredita-se que esta replicação de dados esteja atrelada à um conjunto de fatores como: o link de internet da propriedade que é de $2 \mathrm{MB}$, a lógica de implementação atrelada a capacidade de processamento do Raspberry Pi e 
as configurações de hardware do servidor na nuvem, o qual não estava conseguindo the dar com tanto processamento por possuir apenas $2 \mathrm{~GB}$ de memória RAM.

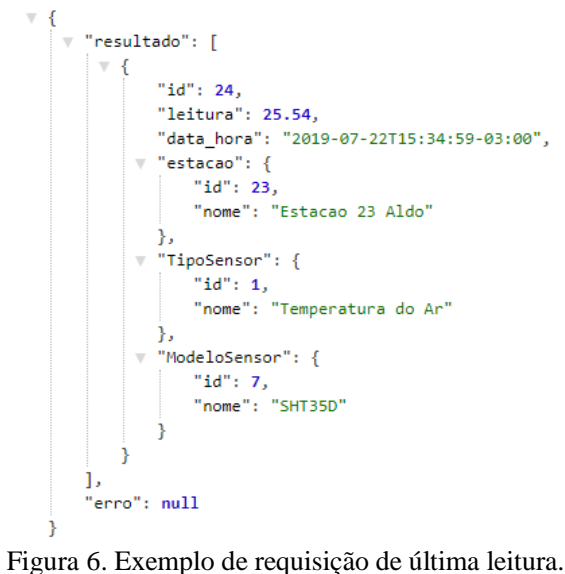

Dessa forma, para otimizar a performance da aplicação buscou-se por alterar a lógica de implementação, todos os dados são armazenados localmente, em caso de sucesso ao ser armazenado na nuvem os dados são excluídos da base local conforme a confirmação de cadastro, reduzindo a quantidade de dados na base local e assim obtendo maior eficiência no processamento dessas informações. Além disso foi aumentado o tamanho da memória RAM do servidor na nuvem de 2 para 4 GB. Estas soluções resolveram os problemas de longos atrasos e de cadastros de dados duplicados.

Foi notado em seguida incorreções na data e hora dos dados que estavam sendo coletados. Por padrão o Raspberry Pi não possui um relógio de tempo real (RTC) integrado, e ele usa a rede ou internet para atualizar suas informações de data e hora por meio do protocolo NTP (Network Time Protocol). Ao cair a conexão com a internet ou energia elétrica, o horário do Raspberry ficava incorreto, dessa forma, para maior confiabilidade foi utilizado um RTC (Real Time Clock) externo, o qual foi acoplado ao mesmo.

Após todos os problemas notados serem corrigidos, foi obtido um bom funcionamento da aplicação com o Raspberry Pi realizando suas tarefas sem problemas.

\section{CONCLUSÃO}

Juntamente com o protocolo MQTT, a arquitetura de computação em névoa, com o Raspberry Pi atuando como broker MQTT sendo implementado em Node.js, se mostrou uma potencial solução para aplicações IoT para a agricultura, principalmente na disponibilidade e na integridade dos dados. Além disso, após realizar a exclusão dos dados armazenados localmente já enviados para a nuvem o Raspberry $\mathrm{Pi}$ se mostrou eficiente, mantendo seu funcionamento constante e estável, atendendo as necessidades deste trabalho. As APIs desenvolvidas proporcionaram aos usuários o acompanhamento remoto das informações disponibilizando serviços para o auxílio nas análises e na tomada de decisão. Como próximos passos está o desenvolvimento de uma plataforma web para o acompanhamento pelo usuário.

\section{AGRADECIMENTOS}

- Parque Tecnológico Itaipu - PTI.

- Centro Latino-americano de Tecnologias Abertas - Celtab.

\section{REFERÊNCIAS}

[1] EMBRAPA. Visão 2030: O futuro da Agricultura Brasileira, DF: Embrapa, 2018. Disponível em: < https://www.embrapa.br/documents/10180/9543845/VisC3A3o+20 30+-+o+futuro+da+agricultura+brasileira/ 2a9a0f27-0ead-991a 8 cbf-af8e89d62829>.

[2] BRASIL, C. PIB e Performance do Agronegócio. Brasília, DF CNA Brasil, 2016., 2016. Disponível em: 〈http://www.cnabrasil.org.br/sites/default/files/sites/default/files/ uploads/02 pib.pdf $\rangle$.

[3] Desa U et al. World population prospects: the 2017 revision. Population division of the department of economic and social affairs of the United Nations Secretariat, New York, 2017.

[4] Brasil G do. Expectativa de vida aumenta em todo o mundo. 2014. Disponível <http://www.brasil.gov.br/saude/2014/12/expectativa-de-vidaaumenta-em-todo-o-mundo>.

[5] Embrapa. Visão 2014-2034: O futuro do desenvolvimento tecnológico da agricultura brasileira: síntese. Brasília, DF: Embrapa, 2014., 2014. Disponível em <https://www.embrapa.br/documents/1024963/1658076/O+Futuro + de+Desenvolvimento+Tecnol\%C3\%B3gico+da+Agricultura+Bra sileira+-+s\%C3\%ADntese.pdf/ddb0a147-234d-47f1-89651959 ef82311d $>$.

[6] ) Zambarda P. 'Internet das Coisas':entenda o conceito e o que muda com a tecnologia. 2014. Disponível em: <http://www.techtudo.com.br/noticias/noticia/2014/08/internetdas-coisas-entenda-o-conceito-e-o-que-muda-comtecnologia.html>.

[7] Fonseca Silveira Massruhá SM. et al. Tecnologias da informação e comunicação e suas relações com a agricultura. Embrapa Informática Agropecuária-Livro científico (ALICE), Brasília, DF Embrapa, 2014.

[8] Ojha T, Misra S, Singh Raghuwanshi N. Wireless sensor networks for agriculture: The state-of-the-art in practice and future challenges. Computers and Electronics in Agriculture, Elsevier, v. 118 , p. 66-84, 2015

[9] Bendre M, Thool, R, Thool V. Big data in precision agriculture: Weather forecasting for future farming. In: IEEE. Next Generation Computing Technologies (NGCT), 2015 1st International Conference on. [S.1.], 2015. p. 744-750.

[10] Bonomi F. et al. Fog computing and its role in the internet of things. In: ACM. Proceedings of the first edition of the MCC workshop on Mobile cloud computing. [S.1.], 2012. p. 13-16.

[11] Stojmenovic I, Wen S. The fog computing paradigm: Scenarios and security issues. In: IEEE. Computer Science and Information Systems (FedCSIS), 2014 Federated Conference on. [S.1.], 2014. p. $1-8$.

[12] Embrapa. Visão 2014-2034: O futuro do desenvolvimento tecnológico da agricultura brasileira: síntese. Brasília, DF: Embrapa, 2014., 2014. Disponível em <https://www.embrapa.br/documents/1024963/1658076/O+Futuro + de+Desenvolvimento+Tecnol\%C3\%B3gico+da+Agricultura+Bra sileira+-+s\%C3\%ADntese.pdf/ddb0a147-234d-47f1-89651959ef82311d>.

[13] SANTOS, H. d. et al. Sistema brasileiro de classifica, $\mathrm{c}^{\text {a ao de solos }}$ Rio de Janeiro: Embrapa Solos, 2006.

[14] CAVIGLIONE, J. H. et al. Cartas clim áticas do estado do paran’a. Londrina: Iapar, v. 1, 2000. 